\title{
Facoemulsificação do primeiro e segundo olho: critérios de indicação e resultados
}

\author{
Phacoemulsification of first and second eye: indication criteria and results
}

\author{
Ana Catarina Delgado de Souza ${ }^{1}$ \\ Rita Flávia Araújo Nicodemos ${ }^{2}$ \\ Catarina Ventura ${ }^{3}$ \\ João Eudes Tavares ${ }^{4}$ \\ Carlos Teixeira Brandt ${ }^{5}$
}

Trabalho realizado na Fundação Altino Ventura e SEOPE Zona Sul.

${ }^{1}$ Pós-graduanda (nível doutorado) em cirurgia pela Universidade Federal de Pernambuco - UFPE - Recife (PE) - Brasil.

${ }^{2}$ Residente do segundo ano de Oftalmologia da Fundação Altino Ventura.

3 Acadêmica do quarto ano de medicina da UFPE - Recife (PE) - Brasil.

${ }^{4}$ Oftalmologista.

${ }^{5}$ Livre docente em cirurgia, coordenador da pós-graduação em cirurgia da UFPE - Recife (PE) - Brasil. Especialista em cirurgia pediátrica.

Endereço para correspondência: Ana Catarina Delgado de Souza. R. Amaro Albino Pimentel, 85 - Recife (PE) CEP 51020-120

E-mail: draanadelgado@hotmail.com

Recebido para publicação em 25.08.2003

Versão revisada recebida em 19.09.2005

Aprovação em 02.12.2005

Nota Editorial: Depois de concluída a análise do artigo sob sigilo editorial e com a anuência do Dr. John Helal Jr. sobre a divulgação de seu nome como revisor, agradecemos sua participação neste processo.

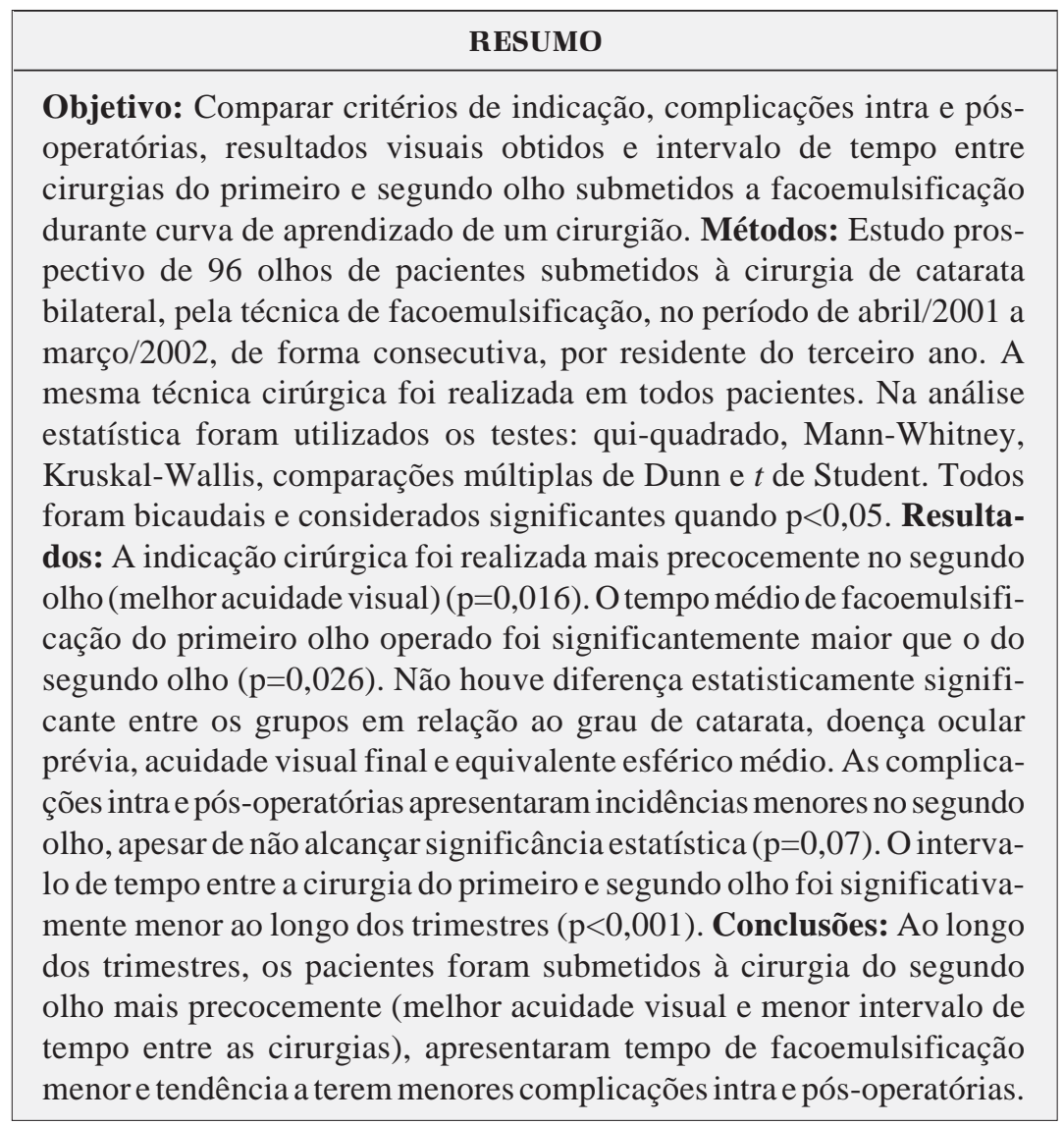

Descritores: Extração de catarata; Facoemulsificação; Implante de lente intra-ocular; Cuidados pós-operatórios; Fatores de tempo

\section{INTRODUCÃ̃}

A facoemulsificação e implante de lente intra-ocular têm aumentado rapidamente em popularidade entre os cirurgiões, inclusive nos países em desenvolvimento, como o Brasil(1-12).

Há diversos motivos que fazem da facoemulsificação a técnica mais utilizada em cirurgias de catarata no mundo, entre eles, pode-se citar a menor incisão, menos trauma ao olho, além de rapidez do ato cirúrgico e da recuperação visual ${ }^{(2,4,6)}$.

Por outro lado, há necessidade de familiarização e aprendizado no manuseio do equipamento para o cirurgião iniciante, o que pode gerar complicações intra-operatórias nos primeiros $\operatorname{casos}^{(1-2,4,6-9,11-12)}$. 
Enquanto os benefícios da cirurgia de catarata no primeiro olho já estão bem estabelecidos e relatados em diversos estudos, os critérios de indicação da cirurgia no segundo olho variam consideravelmente ${ }^{(10)}$.

Acredita-se que haja diferença entre o primeiro e o segundo olho operados em relação à intensidade da catarata, ao grau de acuidade visual e ao número de complicações à medida que o cirurgião vai obtendo mais experiência na técnica ${ }^{(10)}$.

Assim, o objetivo deste estudo foi comparar os critérios de indicação, as complicações intra e pós-operatórias, os resultados visuais obtidos e o intervalo de tempo entre as cirurgias do primeiro e segundo olho, de pacientes submetidos a facoemulsificação durante a curva de aprendizado de um cirurgião iniciante.

\section{MÉTODOS}

Foi realizado estudo prospectivo de pacientes submetidos à cirurgia de catarata bilateral, pela técnica de facoemulsificação, no período de abril de 2001 a março de 2002, de forma consecutiva, por um mesmo residente do terceiro ano, em fase de aprendizado, em serviço de residência em oftalmologia no nordeste do Brasil.

Um formulário pré-estabelecido (contendo perguntas sobre acuidade visual pré e pós-operatórias no primeiro e segundo olho, doenças oculares prévias, classificação das cataratas, tempo de ultra-som utilizado, lente intra-ocular implantada e complicações pós-operatórias imediatas e tardias, em ambos os olhos) foi preenchido, pelo mesmo cirurgião antes da cirurgia, durante a cirurgia e nas visitas pós-operatórias. O período de acompanhamento máximo foi de 11 meses.

Foram excluídos do estudo os pacientes operados apenas uma vez pelo residente em questão, os submetidos a cirurgias combinadas (catarata e glaucoma) e os portadores de catarata traumática ou congênita ou cristalino subluxado.

Todos os pacientes assinaram um termo de consentimento livre e esclarecido declarando-se ciente da pesquisa e permitindo a divulgação de seus dados.

Os pacientes foram submetidos à anestesia peribulbar e à cirurgia pré-estabelecida de facoemulsificação. A técnica consistiu de incisão córneo-escleral tunelizada, capsulorrhexis, hidrodissecção, facoemulsificação pela técnica dividir e conquistar (parâmetros iniciais mantidos, com certa flexibilidade dependendo da catarata, utilizado o aparelho Universal II Alcon), aspiração automatizada de massas corticais e implante de lente intra-ocular de acrílico, de 5,5 mm de zona óptica no saco capsular.

A melhor acuidade visual corrigida pré e pós-operatórias, os tipos de opacificação dos cristalinos e os tempos de ultrasom utilizados em cada cirurgia foram anotados, assim como as complicações intra e pós-operatórias dos dois olhos operados de cada paciente e o intervalo de tempo entre as cirurgias.

$\mathrm{Na}$ análise estatística foi utilizado o teste do qui-quadrado e o de Mann-Whitney para a comparação de variáveis categóricas. Utilizou-se ainda o teste de Kruskal-Wallis e o teste de comparações múltiplas de Dunn. Para observar possíveis diferenças entre as médias das variáveis contínuas foi utilizado o teste " $t$ " de Student. Todos os testes foram bicaudais e considerados significantes quando $\mathrm{p}<0,05$.

\section{RESULTADOS}

Do total de 112 pacientes (160 olhos) submetidos a facoemulsificação por um mesmo residente, no período de um ano, 96 olhos foram operados bilateralmente $(60 \%)$ e incluídos neste estudo. A idade média destes 48 pacientes foi de 63,2 (desvio padrão: 7,6 ) e o gênero feminino representou $58 \%$ da população. Em relação à procedência, 74,8\% moravam na região metropolitana do Recife e 25,2\% eram do interior do Estado. O percentual de aposentados correspondeu a 35,3\%; de trabalhadoras do lar a $41,8 \%$ e de profissionais liberais a $22,9 \%$.

$\mathrm{O}$ olho direito foi o primeiro olho operado em 32 pacientes $(66,7 \%)$.

Nos 96 olhos estudados observou-se pior acuidade visual corrigida no exame pré-operatório do primeiro olho (Gráfico 1). A acuidade visual pré-operatória foi pior ou igual a 20/200 em $45,8 \%$ dos casos no primeiro olho e em $27,1 \%$ no segundo olho. A indicação cirúrgica foi realizada com melhor acuidade visual no segundo olho do que no primeiro olho, com significância estatística $(\mathrm{p}=0,016)$.

Os olhos foram comparados em relação à presença de doenças oculares prévias à cirurgia, tais como: glaucoma, retinopatia hipertensiva e diabética, coroidose mióptica, lesão macular. Não houve diferença estatisticamente significante entre os dois grupos.

A classificação dos tipos de opacificações cristalinianas (grau da catarata, que foi subjetivamente classificada de acordo com o grau de densidade óptica dos núcleos, sendo a

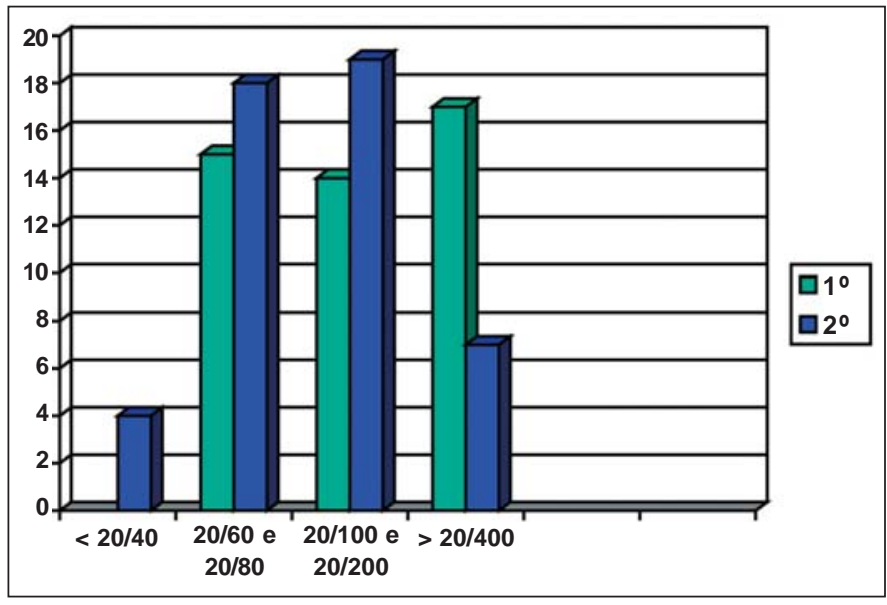

Gráfico 1 - Distribuição das acuidades visuais no pré-operatório em relação ao primeiro e segundo olho operado 
menor densidade descrita como núcleo-esclerose (NS) + e a maior, que impedia a observação das estruturas posteriores ao cristalino, como $\mathrm{NS}++++$, e as opacificações intermediárias correspondendo a graus progressivos de dificuldade de observar as estruturas posteriores ao cristalino, como NS de $++\mathrm{e}$ +++ ) foi similar nos dois grupos, não apresentando diferença estatística, conforme tabela 1 .

Durante a cirurgia o tempo de ultra-som da facoemulsificação foi anotado e posteriormente calculado, observando-se que o tempo médio do primeiro olho operado foi significativamente maior que o tempo de facoemulsificação do segundo olho. $(p=0,026)$ (Tabela 2).

A lente intra-ocular foi implantada na câmara posterior (no saco capsular) em todos os pacientes e apenas nos casos de perda da capsulorrexis a lente intra-ocular foi implantada no sulco capsular.

A presença de complicações intra-operatórias foi comparada entre os dois grupos (primeiro e segundo olho), observando-se que as piores complicações ocorreram no primeiro olho, tais como: ruptura capsular com perda vítrea, descola-

\begin{tabular}{|lrc|}
\hline \multicolumn{3}{|c|}{$\begin{array}{c}\text { Tabela 1. Distribuição do grau de esclerose cristaliniana em rela- } \\
\text { ção ao primeiro e segundo olho operado }\end{array}$} \\
Esclerose cristaliniana & Primeiro olho & Segundo olho \\
NS + ou NS ++ & 27 & 30 \\
NS +++ & 4 & 2 \\
Completa (NS ++++) & 3 & 2 \\
Subcapsular posterior & 13 & 14 \\
Polar & 1 & 0 \\
Total & 48 & 48 \\
NS $=$ núcleo-esclerose & & \\
$p=0,232$ & & \\
\hline
\end{tabular}

\begin{tabular}{|c|c|c|}
\hline & \multicolumn{2}{|c|}{ Tempo de facoemulsificação (min) } \\
\hline & Média & Erro padrão da média \\
\hline Primeiro olho & 2,634 & 0,148 \\
\hline Segundo olho & 2,002 & 0,129 \\
\hline $\begin{array}{l}t=3,214 \\
p=0,0015\end{array}$ & & \\
\hline
\end{tabular}

\begin{tabular}{|lcc|}
\hline \multicolumn{3}{|c|}{$\begin{array}{c}\text { Tabela 3. Distribuição das complicações durante as cirurgias do } \\
\text { primeiro e segundo olho }\end{array}$} \\
Problema cirúrgico & Primeiro olho & Segundo olho \\
Ruptura capsular com vítreo & 2 & - \\
Ruptura capsular sem vítreo & - & 2 \\
Descolamento de Descemet & 1 & - \\
Incisão esgarçada & 1 & 6 \\
Perda da rhexis & 2 & - \\
Sem dificuldades & 42 & 40 \\
p<0,05 & & \\
\hline
\end{tabular}

mento de Descemet e perda da rhexis e este resultado foi estatisticamente significante (Tabela 3 ).

As frequiências das complicações verificadas no período de pós-operatório imediato foram estatisticamente significantes e distribuídas na tabela 4. As complicações mais freqüentes foram edema central e estrias (duas vezes mais freqüente no primeiro olho que no segundo), hipertensão imediata e vítreo na câmara anterior só no primeiro olho e vazamento da incisão só no segundo olho operado (teste exato de Fisher, $\mathrm{p}=0,026)$. Dentre os olhos operados primeiro, foi observada a presença de 34 olhos $(70,8 \%)$ sem qualquer tipo de problema pós-operatório e dentre os segundos olhos, $39(81,2 \%)$ não apresentaram complicações.

O período médio de seguimento foi de 3,7 meses (erro padrão da média: 0,41$)$. O exame pós-operatório mais recente foi utilizado para a análise estatística.

A acuidade visual corrigida, um mês após a cirurgia, foi distribuída segundo a tabela 5. Os segundos olhos apresentaram tendência para melhor acuidade visual, entretanto, este resultado não foi estatisticamente significante. Nove olhos, dentre os 48 operados pela segunda vez, não retornaram para o exame final e refração.

Em relação à refração final, não houve diferença estatisticamente significante entre os equivalentes esféricos médios do primeiro e segundo olho, sendo de: $+0,26$ com desvio padrão de 0,84 e $+0,28$ com desvio padrão de 1,12 , respectivamente.

O resultado do teste de Kruskal-Wallis indica que as distribuições do tempo (em dias) das cirurgias do primeiro e segundo olho foram diferentes entre os trimestres $(\mathrm{p}<0,001)$. Assim, no primeiro trimestre o intervalo de tempo entre as cirurgias do primeiro e segundo olho de cada paciente foi de 132 dias, no

\begin{tabular}{|lcc|}
\hline \multicolumn{3}{|c|}{$\begin{array}{c}\text { Tabela 4. Distribuição das complicações pós-operatórias do pri- } \\
\text { meiro e segundo olho }\end{array}$} \\
Problema pós-operatório & Primeiro olho & Segundo olho \\
Edema central e estrias & 10 & 5 \\
Hipertensão imediata & 2 & - \\
Edema macular cistóide & 1 & - \\
Vítreo na câmara anterior & 1 & - \\
Vazamento na incisão & - & 4 \\
Total & 14 & 9 \\
Teste exato de Fisher $(\mathrm{p}=0,026)$ & & \\
\hline
\end{tabular}

\begin{tabular}{|lcc|}
\hline \multicolumn{3}{|c|}{ Tabela 5. Distribuição dos olhos operados em relação à acuidade } \\
visual final, com correção
\end{tabular}




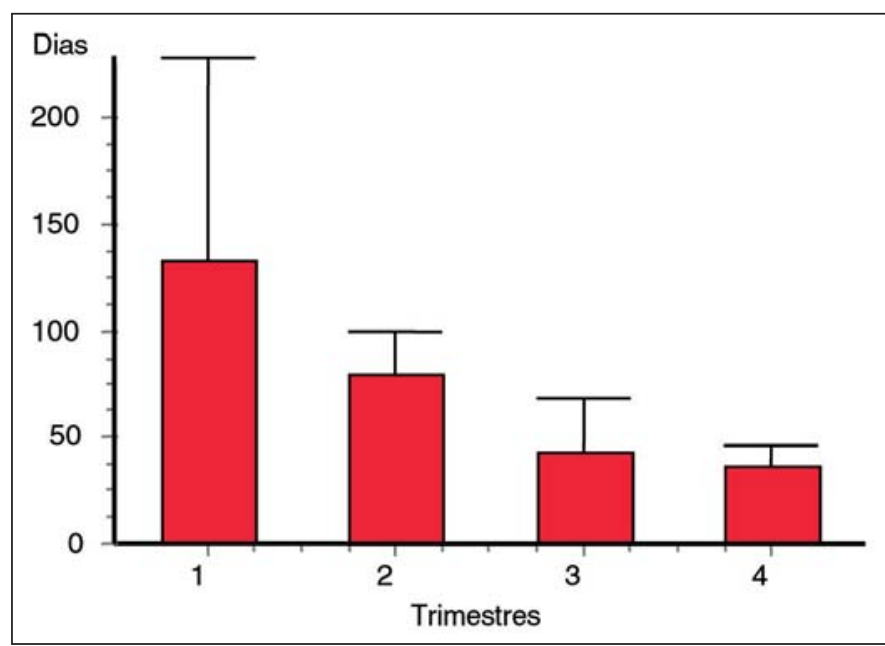

Gráfico 2 - Distribuição do tempo (em dias) entre as cirurgias do primeiro e do segundo olho, de acordo com os trimestres

segundo trimestre de 72 dias, no terceiro de 43 dias e no quarto de 36 dias. Entretanto, ao comparar o primeiro com o segundo trimestre, o segundo com o terceiro e o terceiro com o quarto trimestre, o teste de comparações múltiplas de Dunn revelou diferença estatisticamente significante apenas entre o segundo e o terceiro trimestre $(\mathrm{p}<0,05)$. O gráfico 2 demonstra a distribuição do tempo entre as cirurgias do primeiro e do segundo olho de acordo com os trimestres.

\section{DISCUSSÃO}

A cirurgia de catarata é o procedimento cirúrgico mais freqüente na terceira idade em países desenvolvidos, mesmo quando comparadas com outras cirurgias não oftalmológicas ${ }^{(10)}$.

Entretanto, há grande variação em relação ao tipo de técnica utilizada, aos equipamentos e recursos financeiros disponíveis, às próprias características demográficas da população, etc. Além disto, há significante diferença nos critérios de indicação das cirurgias, dependendo da localização geográfica do país ${ }^{(10)}$. Observou-se que países como Estados Unidos, Canadá e Dinamarca operam seus pacientes com acuidade visual corrigida pré-operatória muito melhor que em outros países como por exemplo Espanha (que apresentou baixa acuidade visual pré-operatória: 20/200 ou pior em 59,1\% dos ca$\operatorname{sos})^{(13)}$. Neste estudo esta acuidade visual pré-operatória também foi melhor que a verificada na Espanha (45,8\% dos casos no primeiro olho e $27,1 \%$ no segundo).

Adicionalmente a esta heterogenicidade nos critérios de indicação da cirurgia de catarata, também há variação entre os dois olhos dos pacientes: demonstrou-se neste estudo que a indicação cirúrgica do segundo olho é realizada quando o paciente tem acuidade visual melhor do que a que determinou a indicação cirúrgica para o primeiro olho. Este fato pode ser explicado pela maior exigência visual do paciente, após perce- ber a melhora da função visual do primeiro olho. Sabe-se que os benefícios para operar o primeiro olho em relação à acuidade visual e à função visual são bem estabelecidos, enquanto o mesmo não ocorre em relação ao segundo olho ${ }^{(10)}$. Por outro lado, a melhora comprovada da função visual e a binocularidade (estereopsia) prevalecem quando se opera bilateralmente ${ }^{(10)}$.

Houve registro de precocidade na indicação na cirurgia não só em relação à acuidade visual, como também em relação ao tempo entre as duas cirurgias. Quando se comparou o intervalo de tempo entre as cirurgias e os trimestres do ano em que foram realizadas, observou-se uma tendência do cirurgião a indicar a segunda cirurgia cada vez mais cedo (tempo médio do primeiro trimestre foi de 132 dias entre as cirurgias e do quarto trimestre foi de 36 dias).

Um fator que pode interferir na antecipação para realizar a segunda cirurgia pode ser a autoconfiança do cirurgião, ao longo da curva de aprendizado, que cada vez mais se sente seguro e habilitado para indicar a cirurgia após poucos dias da primeira. Outro fator importante que deve ser considerado é a precocidade da indicação na tentativa de evitar repetir os exames pré-operatórios, que normalmente vencem com três meses. Desta forma, o paciente se propõe a realizar a segunda cirurgia dentro deste prazo.

Estudo espanhol demonstrou, diferentemente destes resultados, um longo tempo entre as duas cirurgias, com média de 18,8 meses $^{(10)}$. Os autores espanhóis sugerem que 20 a $40 \%$ dos pacientes vão se submeter à cirurgia do segundo olho dentro de 12 meses após o primeiro olho, discordando dos resultados obtidos nesta investigação onde $60 \%$ foram operados bilateralmente em um ano de seguimento ${ }^{(10)}$.

Os autores reconhecem que a pequena amostra de pacientes deste estudo não demonstrou poder suficiente para provar significância estatística em relação às complicações intra e pós-operatórias e à acuidade visual final entre o primeiro e o segundo olho. Desta forma, a análise destes dados estão sendo continuadas seguindo o mesmo protocolo, a fim de aumentar a casuística e a significância do estudo.

Apesar da classificação dos tipos de opacificações do cristalino ter sido similar nos dois grupos deste estudo, acredita-se que cataratas mais densas têm maior risco de complicações e são as operadas primeiro ${ }^{(5-6)}$. O maior tempo de ultrasom utilizado na facoemulsificação do primeiro olho, além de significar menor experiência do cirurgião no início do treinamento, também sugere que a catarata estava mais madura que a do segundo olho.

\section{CONCLUSÕES}

Neste estudo, os pacientes foram submetidos à cirurgia do segundo olho mais precocemente (melhor acuidade visual e menor intervalo de tempo entre as duas cirurgias), apresentaram tempo de facoemulsificação menor e tendência a terem menores complicações intra e pós-operatórias. 


\section{ABSTRACT}

Purpose: To compare indication criteria, intra- and postoperative complications, visual outcomes and time interval between the first and second eye surgery (phacoemulsification). Methods: Prospective study was done in 96 eyes of patients who underwent bilateral consecutive phacoemulsification surgery, performed by a third-year ophthalmology resident. An established protocol was filled out before, during and after the surgery. The same surgical technique was used. Chi-squared, Mann-Whitney, Kruskal-Wallis, Dunn multiple comparison and Student's $t$ tests were used for statistical analysis. Bicaudal tests were used in all situations. $p<0.05$ rejected the null hypothesis. Results: The indication for the second eye surgery was done earlier and with best correct visual acuity when compared with the first eye $(\mathrm{p}=0.016)$. The mean phacoemulsification ultrasound time (US time) of the first eye was significantly higher $(\mathrm{p}=0.026)$. There were no statistically significant differences between the two groups in relation to type of cataract, previous intraocular diseases, final visual acuity and mean spherical equivalent. The incidence of intra- and postoperative complications was smaller in the second eye, but was not statistically significant $(\mathrm{p}=0.07)$. Time interval between the first and second eye surgery was significantly lower along the trimesters. Conclusion: The patients underwent the second eye phacoemulsification surgery earlier (with better visual acuity and shorter time interval), had a shorter phacoemulsification time and tendency to be followed by less intraand postoperative complications.

Keywords: Cataract extraction; Phacoemulsification; Lens implantation, intraocular; Postoperative care; Time factors

\section{REFERÊNCIAS}

1. Cruz OA, Wallace GW, Gay CA, Matoba AY, Koch DD. Visual results and complications of phacoemulsification with intraocular lens implantation performed by ophthalmology residents. Ophthalmology. 1992;99(3):448-52. Comment in: Ophthalmology. 1992;99(8):1181

2. Lambert LC, Occhiutto ML, Paparelli CM, Kniggendorf S, Akaishi L, Mendonça $\mathrm{BD}$ et al. Resultados visuais e incidência de complicações em facoemulsificação com LIO por residentes. Rev Bras Oftalmol 1997;56(12):953-6.

3. Freitas JAH, Soranz Filho JE, Rovigotti Junior V, Barbosa ML, Atique D. Extração extracapsular do cristalino: experiência do ensino no curso de residência médica. Rev Bras Oftalmol. 1997;56(12):957-61.

4. Rodrigues Martín J, Baudet Naveros B, Martin Barrera F, Abreu Reyes JA. Cirugía de la catarata: de la extracción extracapsular a la facoemulsificación. Evolución y resultados obtenidos por un residente de tercer año. Arch Soc Canar Oftalmol. 2000;11:1-4

5. Martin KR, Burton RL. The phacoemulsification learning curve: per-operative complications in the first 3000 cases of experienced surgeon. Eye. 2000;14(Pt2): 190-5.

6. Domingues FGP, Crema AS, Yamane Y. Complicações intra-operatórias da facoemulsificação durante a residência médica. Rev Bras Oftalmol. 2000;59(4): 275-9.

7. Badoza DA, Jure T, Zunino LA, Argento CJ. State-of-the-art phacoemulsification performed by residents in Buenos Aires, Argentina. J Cataract Refract Surg. 1999;25(12):1651-5. Comment in: J Cataract Refract Surg. 2000;26(6):794-6.

8. Tarbet KJ, Mamalis N, Theurer J, Jones BD, Olson RJ. Complications and results of phacoemulsification performed by residents. J Cataract Refract Surg. 1995;21(6):661-5.

9. Corey RP, Olson RJ. Surgical outcomes of cataract extractions performed by residents using phacoemulsification. J Cataract Refract Surg. 1998;24(1):66-72. Comment in: J Cataract Refract Surg. 1998;24(6):727-9.

10. Stark WJ, Worthen DM, Holladay JT, Bath PE, Jacobs ME, Murray GC, et al. The FDA report on intraocular lenses. Aust J Ophthalmol. 1984;12(1):61-9.

11. Blomquist PH, Rugwani RM. Visual outcomes after vitreous loss during cataract surgery performed by residents. J Cataract Refract Surg. 2002;28(5):847-52.

12. Albanis CV, Dwyer MA, Ernest JT. Outcomes of extracapsular cataracts extraction and phacoemulsification performed in a university training program. Ophthalmic Surg Lasers. 1998;29(8):643-8.

13. Alonso J, Espallargues M, Andersen TF, Cassard SD, Dunn E, Bernth-Petersen $\mathrm{P}$, et al. International applicality of the VF-14. An index of visual function in patients with cataracts. Ophthalmology. 1997;104(5):799-807.

\section{Congresso Internacional da Sociedade Brasileira de Oftalmologia}

20 a 22 de Julho de 2006

Holel Clóríc.

Rio de Janeiro - RJ 